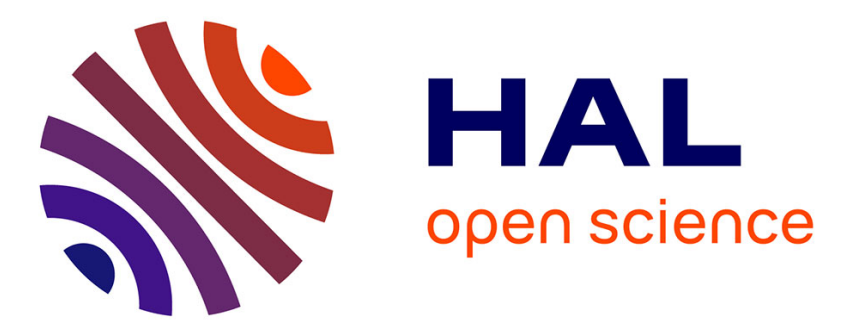

\title{
Diagnosis of injection-molded weld lines in ABS thermoplastic by polarized terahertz reflective imaging
}

Min Zhai, Esam T. Ahmed Mohamed, A. Locquet, G. Schneider, R. Kalmar, M. Fendler, Nico F F Declercq, D.S. S Citrin

\section{- To cite this version:}

Min Zhai, Esam T. Ahmed Mohamed, A. Locquet, G. Schneider, R. Kalmar, et al.. Diagnosis of injection-molded weld lines in ABS thermoplastic by polarized terahertz reflective imaging. NDT \& E International, 2021, 122, pp.102497. 10.1016/j.ndteint.2021.102497 hal-03419659

\section{HAL Id: hal-03419659 \\ https://hal.science/hal-03419659}

Submitted on 8 Nov 2021

HAL is a multi-disciplinary open access archive for the deposit and dissemination of scientific research documents, whether they are published or not. The documents may come from teaching and research institutions in France or abroad, or from public or private research centers.
L'archive ouverte pluridisciplinaire HAL, est destinée au dépôt et à la diffusion de documents scientifiques de niveau recherche, publiés ou non, émanant des établissements d'enseignement et de recherche français ou étrangers, des laboratoires publics ou privés. 


\section{Diagnosis of injection-molded weld lines in ABS thermoplastic by polarized terahertz reflective imaging}

Min Zhai, ${ }^{1,2}$ Esam T. Ahmed Mohamed, ${ }^{1,3}$ Alexandre Locquet, ${ }^{1,2}$ G. Schneider, ${ }^{4}$ R. Kalmar, ${ }^{4}$ M. Fendler, ${ }^{4}$ N. F. Declercq, ${ }^{1,3}$ and D.S. Citrin ${ }^{1,2, \bigotimes}$

${ }^{1}$ Georgia Tech-CNRS IRL2958, Georgia Tech Lorraine, 2 Rue Marconi, 57070 Metz, France

${ }^{2}$ School of Electrical and Computer Engineering, Georgia Institute of Technology, Atlanta, Georgia, 30332-0250 USA

${ }^{3}$ School of Mechanical Engineering. Georgia Institute of Technology, Atlanta, Georgia, 30332 USA

${ }^{4}$ CEA Tech, 5 Rue Marconi, Bâtiment Austrasie, 57070 Metz, France.

$\triangle$ Corresponding author: david.citrin@ece.gatech.edu

Tel: +1 404 717-3085 


\section{HIGHLIGHT}

- Terahertz (THz) reflective imaging and scanning acoustic microscopy (SAM) were applied to characterize the injection-molded weld lines in an ABS electrical receptacle plate.

- V-shaped notches are found in both SAM and $\mathrm{THz}$ results.

- Spatially dependent $\mathrm{THz}$ birefringence resulting from the fountain flow in the melt is highly correlated with the weld-line location.

- THz imaging, in conjunction with SAM, serves as an effective approach for quality control of injectionmolded thermoplastics in a nondestructive fashion. 


\section{ABSTRACT}

We carry out a nondestructive study of injection-mold weld lines in an acrylonitrile butadiene styrene (ABS) thermoplastic electrical receptacle plate. Terahertz techniques show morphological features at the weld lines and reveal terahertz birefringence properties localized about the welds, indicating frozen-in molecular orientation and stress proximate to the welds. Surface acoustic microscopy corroborates the morphological features at the welds shown by terahertz imaging and also reveals anomalous mechanical properties at the weld lines. These results indicate that $\mathrm{THz}$-based techniques provide nondestructive reliability assessment and quality control for injection-molded thermoplastic products.

Index Terms - Nondestructive testing; Terahertz radiation; Ultrasonic imaging; Injectionmolded weld lines; Birefringence. 


\section{INTRODUCTION}

Injection molding is the most commonly used method to manufacture plastic parts, providing design flexibility and high-output production. Stagnating weld lines, a common defect in injection-molded thermoplastic products, form where two separate melt fronts impinge headon after which there is no subsequent flow, as shown schematically in Fig. 1(a). Of practical concern, such surface defects degrade the mechanical properties and are places where fracture or other failure is likely to occur [1-5]. Generally, the origin of the reduced mechanical strength of weld lines are molecular entanglement, the formation of V-notches at the weld surface, as well as the presentation of contamination at the weld interfaces. In order to guarantee the quality of fabricated injection-molded thermoplastic products, it is important to understand the geometry, morphology, and nature of melt flow at weld lines.

Injection-mold weld lines may or may not be easily identified by the naked eye or straightforward optical microscopy. The most common method for weld-line characterization is mechanical testing by means of a tensile or bend stage [6,7]. The relative weld-line strength is defined as the ratio of the strength of specimens with a weld line and a comparable sample with the same geometry but without the weld line, and this measure mainly depends on the molding conditions, such as melt temperature, mold temperature, and injection pressure [8]. Even though these approaches provide qualitative and quantitative information, they are restricted to certain geometries and, moreover, are destructive. X-ray computed tomography can provide three-dimensional reconstruction of the microstructure of weld joints, though x-ray induced damage may ensue after the measurement $[9,10]$; moreover, x-ray measurement may engender significant health risk and is therefore not suitable for practical application. Surface probes, such as atomic-force microscopy, suffer from limited scan area, relatively long scan duration, and may fail to provide sufficient information [11]. For scanning electron microscopy, the sputtering a conductive gold layer limits the reuse of sample for other tests $[8,12]$. As for 
conventional far-field ultrasound testing, the frequent requirement of a coupling medium may make the approach cumbersome and acoustic waves at the requisite frequencies $(\sim 10 \mathrm{MHz})$ for high resolution may suffer from high attenuation in thermoplastics [9,13]. Therefore, alternative nondestructive evaluation (NDE) techniques for understanding thermoplastic injection-weld lines and for the monitoring of components in production are of interest.

In addition to surface morphological features at injection-mold weld lines in thermoplastic specimens, internal features might also be used for the NDE of such weld lines. For example, anisotropy might also be induced in the weld-line vicinity as a consequence of the rheological state of the melt flow. Typically, most amorphous polymer plastics at equilibrium are inherently isotropic and exhibit no birefringence. However, due to fountain flow [14,15], a combination of shear and elongational flows, the melt-flow fronts are subjected to shearing flow during the mold filling process, which leads to increase of elasticity and preferential molecular orientation proximate to the weld line, and the formation of complex anisotropic structure at the weld line of the injection molded parts after cooling [16]. The solidification of melt flows leads to frozenin anisotropic stress and molecular orientation in injection-molded plastics, which in turn can induce the reduction of the mechanical strength when the forces are applied perpendicular to the weld line $[17,18]$. The degree of orientations can be quantified by the birefringence index. In Ref. [19], optical birefringence distribution along the flow direction of injection-molded thermoplastic specimens has been investigated through a photo-elasticity method. The influences of the temperature distribution and shear-rate field within various areas near a weld line during the injection molding affect birefringence have been studied using Z-MOLD analysis software, which is an integrated computational simulation suite for injection-molded products [20]. The local anisotropic stresses specifically proximate to injection weld lines have not, however, been as extensively studied experimentally. Such spatially dependent anisotropy could also provide a contrast mechanism for various NDE techniques to validate and 
characterize the presence of injection-mold weld lines even when such weld lines are not easily identified visually. In particular, detecting the weld-line location, understanding their spatial extent, and gaining insight into local anisotropy could be used to optimize the manufacturing processes that avoid the reduction of weld strength of fabricated injection-molded thermoplastic products.

Terahertz (THz) imaging has attracted interest for the NDE of non-polar and polar plastic materials and components [17,18,21-24]. $\mathrm{THz}$ waves are electromagnetic radiation with frequencies from $100 \mathrm{GHz}$ to $10 \mathrm{THz}$ range, which can penetrate many nonelectrically conductive materials with high spatial resolution that are otherwise opaque to visible light as well as near-infrared light or present little contrast in x-ray imaging, and provide means to reveal the structural and spectroscopic information of such materials in a nondestructive fashion [25]. Due to the low energy of THz photons and the low powers employed, they present minimal health risks [26]. Because of these characteristics, $\mathrm{THz}$ techniques merit exploration to understand weld-line formation and for in-line inspection and quality control of injectionmolded thermoplastic components.

In our previous work presented in Ref. [27], the acoustic properties near injection-weld lines were investigated by comparing the velocity of Rayleigh surface acoustic waves (RSAW) in locations proximate to and distant from the weld lines. A discrepancy of about $100 \mathrm{~m} / \mathrm{s}$ in the velocity of RSAWs between the locations was found, indicating anisotropic propagation of differently polarized ultrasonic waves. The micro-elastic heterogeneity near injection-weld lines might induce the THz birefringence. The morphological deformation was also observed using $\mathrm{THz}$ reflective imaging and presents an excellent agreement with scanning acoustic microscopy (SAM). In this study, we apply polarization-resolved $\mathrm{THz}$ imaging to characterize the spatially dependent $\mathrm{THz}$ birefringence due to the anisotropy near weld lines, and verify the interpretation of the SAM results in Ref. [27]. By these means we locate injection-mold weld 
lines in an Acrylonitrile Butadiene Styrene (ABS) thermoplastic electrical receptacle plate in a nondestructive manner and characterize the associated $\mathrm{THz}$ electromagnetic, acoustomechanical, and morphological features. The spatial distribution of $\mathrm{THz}$ birefringence was also investigated and related to the variation of melt-flow direction as well as the mechanical properties.

In Sec. 2 we present the sample and outline the experimental technique. Section 3 presents the results, while in Sec. 4 we conclude.

\section{EXPERIMENTAL TECHNIQUES}

\subsection{Sample Description}

Figure 1 (b) and (c) show the injection-molded component, with dimensions $156 \mathrm{~mm} \times 85 \mathrm{~mm}$ $\times 4 \mathrm{~mm}$ (at rim), studied here. It is an ABS thermoplastic electrical receptacle plate produced by Schneider Electric (S520704 Odace 2-gang electrical receptacle plate). ABS is a common thermoplastic used in a host of electrical, decorative, structural, medical, and consumer applications due to its impact resistance, toughness and chemical stability. Besides, ABS is amorphous and may have a range of compositions; the specific values for the component studied here are unavailable. In general, ABS is a two-phase material mixing by a styreneacrylonitrile copolymer with polybutadiene. A two-gate injection mold was used to fabricate this component showing symmetry along the long axis. The processing condition of the injection molded parts, including melting temperature, melting speed, and mold temperature, are unavailable. The injection-mold line runs horizontally through the three rectangles indicated in Fig. 1(b), which shows the front of the plate (decorative side). Figure 1(c) shows the back of the plate (facing the electrical box). 


\subsection{Terahertz imaging}

THz measurements in this work are carried out using a pulsed, broadband $\mathrm{THz}$ time-domain system from TeraView Ltd, namely a TPS Spectra 3000, shown schematically in Fig. 2(a). The GaAs photoconductive antenna is excited by an Er-doped fibre laser which emits sub-100 fs near infrared pulses at $780 \mathrm{~nm}$ at a repetition rate of $100 \mathrm{MHz}$ to produce roughly single circle THz pulses with bandwidth extending from $60 \mathrm{GHz}$ to $3 \mathrm{THz}$. Due to the low power of $\mathrm{THz}$ radiation used for the measurement, negligible thermal strain is induced in the sample. $\mathrm{THz}$ imaging is performed in reflection mode at almost normal incidence. Excitation of rotational modes of atmospheric $\mathrm{H}_{2} \mathrm{O}$ causes ringing after the main $\mathrm{THz}$ pulse and sharp absorption lines in the power spectrum. Such undesirable effects are eliminated by purging with dry $\mathrm{N}_{2}$ in the propagation path $[28,29]$. Balancing the computational cost, the limitation of our experimental setup, and the spatial resolution, the sample is raster-scanned by a set of motorized stages moving in the $x$ - and $y$-directions with transverse step size (pixel size) of $200 \mu \mathrm{m}$, and each recorded reflected THz pulse contains 4096 data points. The recorded signal is averaged over 10 shots per pixel to reduce the effect of noise. Due to our choice of THz optics, the depth of focus is $\sim 7 \mathrm{~mm}$ and thus the beam waist is approximately constant over the depth of the sample. The large depth of focus minimizes the impact of focusing error and the Guoy phase. The transverse resolution is $\sim 300 \mu \mathrm{m}$, though the beam characteristics in both the axial and transverse directions are expected to depend on wavelength within the experimental bandwidth. Our measurements are carried out in the time domain in reflection. That is, we measure the reflected $\mathrm{THz}$ signal in time acquiring both amplitude and phase information. The beam can be rastered along a line or area of the sample to build up a line scan or image. Experiments are carried out in a polarization-resolved fashion. 


\subsection{Scanning Acoustic Microscopy}

The acoustic microscope utilized in this study (ELSAM, Ernst-Leitz Scanning Acoustic Microscope; PVA TePla Analytical Systems, GMBH, Deutschordenstrasse 38, 73463 Westhausen, Germany) operates in reflection mode, and is shown schematically in Fig. 2(b). A piezoelectric transducer mounted on the back surface of an acoustic lens receives a short RF pulse (approximately 10-30 ns in duration) and converts it into mechanical waves. The acoustic waves propagate through the sapphire rod and are focused sharply by the spherical cavity of the lens into a diffraction limited point in the interface between a coupling fluid and the sample. The signal reflected at the different interfaces is collected back by the same transducer and converted into an electric signal. An image is then formed by scanning in two dimensions and processing the reflected signal into a greyscale map. Figure 2(b) illustrates the basic principle in SAM.

The contrast in SAM is based on the acousto-mechanical properties of the sample. The amplitude reflection coefficient from medium $i$ to $j$ is

$$
R=\frac{Z_{i}-Z_{j}}{Z_{i}+Z_{j}}
$$

where, $Z_{i, j}=\rho_{i, j} V_{i, j} / \cos \theta$ is the acoustic impedance of medium $i, j$ of mass density $\rho_{i, j}$ and acoustic velocity $V_{i, j}$, with $\theta$ the angle of incidence. $V_{i, j}$ is related to the bulk and shear moduli, and consequently to Young's modulus of elasticity for a material with a constant Poisson's ratio [30].

\section{RESULTS AND DISCUSSION}

\section{1. $\mathrm{THz}$ Characterization}

We are unable to sense the weld-line by touch or to see it with the unaided eye. We therefore imaged the electrical receptacle plate in the weld-line region at $50 \times$ by optical microscopy [Axio Scope.A1 Microscope (Zeiss)]. No weld-line defect was easily identifiable under the 
optical microscope. Moreover, due to the irregular shape, it is hard to achieve a good focus on the surface of the thermoplastic specimen without cutting. We also conducted tensile testing to failure on nominally identical receptacle plates. In most cases, macroscopic fracture initiated at the same positions, i.e., the weld lines, which are known to be common sources of mechanical failure in injection-molded thermoplastic components.

$\mathrm{THz}$ imaging in reflection mode from the front (decorative) side was conducted first to characterize the surface morphology of injection-molded plastic samples. Owing to the symmetry of the receptacle plate along the horizontal axis and the location of the input ports for the injected flows, the weld lines are expected to be located horizontally within the regions of interest (ROI) marked I, II, and III in Fig. 1(b). The dimension of the ROI we investigated is $1 \mathrm{~cm}$ and $2 \mathrm{~cm}$, respectively. Based on the set experimental parameters as well as scanning area, the duration can be calculated, which is $\sim 2$ hours. By monitoring the arrival time of the peak of the reflected $\mathrm{THz}$ signal as a function of position across the ROI's, we obtain a measure to the surface morphology of the front side. That is, if the peak of the reflected signal arrives later in some area, the surface there is indented compared with surrounding areas due to the additional time-of-flight involved to receive the main feature of the reflected signal. In other words, the additional time delay $\tau$ in receiving the signal gives the relative local depth of the surface $d=$ $c \times \tau / 2$. Here, $c$ is the in vaccuo speed of light and the factor of 2 in the denominator accounts for the additional time for the peak signal's round trip.

C-scans (two-dimensional presentation of components) based on this contrast mechanism, corresponding to the air/injection-molded plastic sample interface, are plotted in Fig. 3. The THz polarization was first set to $0^{\circ}$, defined to be parallel to the horizontal weld line. In all three ROIs we detect a V-shaped notch at the presumed weld-line locations, which is perpendicular to the direction of melt flows meeting at the center of the thermoplastic electrical receptacle plate. The surface morphology of the V-shaped notches can be estimated based on the arrival 
time of echo reflected from the surface of thermoplastic as discussed above. We obtain values for the notch width and depth of $\sim 400 \mu \mathrm{m}$ and $\sim 10 \mu \mathrm{m}$, respectively, based on the $\mathrm{THz} \mathrm{C}$ scans of Fig. 3. The width and depth of notch extracted from the THz measurement is limited by the applied experimental parameters, such as the transverse resolution $(\sim 300 \mu \mathrm{m})$, the spatial step size in rastering the sample $(200 \mu \mathrm{m})$, and the sampling frequency $(\sim 0.0116 \mathrm{ps})$. The use of SAM which can provide $\sim 3.5 \mu \mathrm{m}$ transverse resolution at center frequency at $420 \mathrm{MHz}$ to validate the $\mathrm{THz}$ measurements is discussed below. Here we note that we attempted atomicforce microscopy to verify the surface morphology, but due to the irregular sample geometry (of the back of the plate) and its size, vibrations rendered these measurements fruitless.

There are several plausible sources for the formation of V-shaped notches at injection-mold weld lines. One is poor bonding due to insufficient time prior to solidification for the polymer molecules to diffuse across the interface. Another is frozen-in molecule orientation which is preferentially parallel to melt flow which ends up being parallel to the weld line. Others include anisotropic shrinkage near the weld during cooling [31], air entrapped at the interface between the flow fronts [32], the poorly bonded region near the surface in combination with the large shrinkage due to the high molecular orientation [33]. Because of the absence of detailed information on the fabrication procedure of this thermoplastic receptacle plate, we can only provide these plausible, though tentative, explanations. The measurements discussed above of the surface morphology show negligible dependence on the THz polarization. The bulk internal properties of the thermoplastic, however, show significant polarization dependence as is now discussed.

Broadly speaking, optical birefringence is known to provide a sensitive probe of molecular orientation and residual stress in amorphous polymer injection plastics $[17,19,34,35]$, and can also be treated as a microscopic structural features as well as the anisotropic manifestation of injection-molded thermoplastic products. The component under study is optically opaque, so 
$\mathrm{THz}$ birefringence arises as a candidate to probe the internal structure near the weld lines. Figure 4 shows typical reflected $\mathrm{THz}$ signals close to the weld for $\mathrm{THz}$ polarization perpendicular (red) and parallel (black) to the weld line. Each reflected signal shows a pronounced first echo (near optical delay $10 \mathrm{ps)} \mathrm{from} \mathrm{the} \mathrm{air/plastic} \mathrm{interface} \mathrm{(decorative} \mathrm{side)}$ followed by ringing (due to noise, atmospheric $\mathrm{H}_{2} \mathrm{O}$, and after-pulses in the photoconductive emitter). At $\sim 35$ ps we observe a weaker echo originating from the back plastic/air interface. The sign of the second echo is negative as expected since the refractive index of air is less than that of the plastic. The inset shows a slight difference in arrival time of the second echo for the two polarizations with the echo arriving $\Delta t$ earlier for perpendicular $(\perp)$ polarization with respect to the case of parallel (II) polarization, indicating that the refractive index $n_{\perp}$ for the former polarization is slightly larger than that $n_{\|}$for the latter polarization. The polarizationdependent refractive index is attributed to the variation of local density and melt flow direction due to the fountain flow (from perpendicular to the weld line to parallel to the weld line, shown in Fig. 1(a)) [36]. The value of refractive indices at different polarizations can be obtained from $w=c T_{i} /\left(2 n_{i}\right)$ where $w$ is the thickness of the receptacle plate at the location measured, $c$ is the in vaccuo speed of light, $T_{i}$ is the time delay between the first and second echo in the reflected signal for polarization $i=\perp, \|, n_{i}$ is the refractive index for polarization $i$, and the factor of 2 in the denominator is due to the fact that the reflected signal passes through the plate thickness $w$ twice. Caliper measurement of the plate thickness at the location measured on the weld line gives $w=1.6 \mathrm{~mm}$. We thus find the values $n_{\perp}=1.7277$ and $n_{\|}=1.693$ giving $\mathrm{THz}$ birefringence $\Delta n=\left|n_{\|}-n_{\perp}\right|=0.035$. We neglect the effects of THz dispersion, which are relatively weak in thermoplastics [37]. In general, the variation of refractive index for different polarizations might result from the change with position of the preferential molecular orientation. During mold filling, molecular chains at the melt front are stretched parallel to the weld line as a result of this flow, and the initial orientation begins to freeze in from the surface. 
Because the molecules in the interior have more time to relax their orientations before they are frozen, the molecular orientation might be partially relaxed during solidification. The remaining frozen-in molecular orientation affects the tensile strength of the weld line as well as the $\mathrm{THz}$ birefringence [2]. Moreover, the variation of the mechanical density near the weld line will also influence the refractive index $n$, even though it is difficult to quantify the spatial dependence [27].

To study the birefringence $\Delta n$ and its spatial dependence in more detail, we measure the refractive index $n$ as a function of polarization angle $\theta$ with respect to the of weld-line direction in ROI I. Because the $\mathrm{THz}$ polarization angle is fixed, the $\mathrm{THz}$ time-domain waveforms according to different $\theta$ are measured by rotating the injection-molded component placed on the focal plane. Figure 5 shows the polarization dependence of $n$ at the weld line where the dotted black curve is a fit of the data to $A \times \sin \left(2 \theta+\varphi_{1}\right)+B$; Root Mean Square Error (RMSE), a standard approach of evaluating the residuals distribution, is calculated to assess the fit quantitatively. A small RMSE, which is 0.003787 , shows an excellent correlation between the measured values and fitting results. The polarization-dependent refractive index $n$ achieves its minimum at $\theta=\sim 0^{\circ}$ corresponding to $n_{\|}$and its maximum at $\sim \pm 90^{\circ}$ corresponding to $n_{\perp}$. We then investigated $\Delta n$ in ROI I, but $\sim 1 \mathrm{~mm}$ away from the weld line as presented in Fig. 6 . In this case $\Delta n=0.0087$, less than half that on the weld line itself, demonstrating that the degree of anisotropy decreases moving away from the weld line. Similar results (not shown) are found in ROIs II and III.

The tentative explanation of these results is that at the weld line, the flows result in a preferred frozen-in molecular orientation and/or stress field parallel to the weld line. As one moves away from the weld line over a certain length scale, $\Delta n$ tends to be zero as the ABS tends toward an isotropic state [38,39]. We expect this distance depends on the flow and cooling rates as well as the composition. Such spatially dependent anisotropy and morphological features could 
provide contrast mechanisms for various NDE techniques to detect the presence of and characterize injection-mold weld lines.

To verify that indeed this pronounced variation in $\Delta n$ is connected with the weld line, in Fig. 7 we plot $\Delta n$ as a function of position along a section perpendicular to the weld in ROI I with $d=6 \mathrm{~mm}$ corresponding to the weld. A Gaussian fit to the data is shown as a guide to the eye. We see that the enhanced $\mathrm{THz}$ birefringence is localized around the weld gradually decreasing on the length scale of $2 \mathrm{~mm}$ with distance from the weld. The weld lines in ROIS II and III show similar behaviors.

\subsection{SAM Characterization}

Another NDE technique that can provide insight into the weld line is SAM. In addition, we wish to validate our interpretation of the THz results by independent measurements. SAM is of interest to ascertain the effects of the welding process on the visco-elastic properties and to visualize the micro-elastic spatial variations near the weld lines. SAM is an established choice for the quality control of industrial products and for health monitoring, quality control, and troubleshooting [40-43]. It enables the non-invasive and non-destructive assessment of the micro-elastic properties of objects with a high spatial resolution when a focusing piezoelectric transducer (acoustic lens) is utilized at ambient temperature using water as a coupling fluid [44]. For the work presented here, SAM with a center frequency of $420 \mathrm{MHz}$ was employed to achieve sufficient resolution to probe anomalous acousto-mechanical and morphological behavior close to the weld lines. The lateral resolution of SAM that operates at a center frequency of $420 \mathrm{MHz}$ is close to $3.5 \mu \mathrm{m}$ at room temperature, which is much smaller than the spatial step size we employed $(100 \mu \mathrm{m})$. In that sense SAM has a much higher spatial resolution than THz-based approach. SAM also allows us to overcome resolution difficulties that arise in standard far-field ultrasonic approaches. 
From the SAM image of ROI shown in Fig. 8(a), a weld line with heterogeneity in the microstructure with respect to the mechanical properties and flow patterns is detectable, as was also reported in Ref. [27]. The estimated width of the weld line is $\sim 500 \mu \mathrm{m}$. In Fig. 8(b), morphological deformations on the surface of the mold about $1 \mathrm{~mm}$ distance from the weld line are observed at the weld, which may be linked to the thermal effects followed by solidification of the mold during the injection molding process. Similar structures are also found in regions of interest; ROIs II and III.

In addition to the observation of the morphological features at the weld line, SAM imaging offers contrast depending on spatial variation in the viscoelastic and mechanical properties, hence, the complementary nature of this technique with $\mathrm{THz}$ imaging. In the magnitudecontrast acoustic microscopy images shown in Fig. 8(b), surface heterogeneity, mainly associated with the reflectivity but also to some degree with the attenuation, can be assessed. Variations in ultrasonic reflectivity are connected to local variations in the mass density, elastic moduli (bulk compression, shear, and Young's), or in both. Variations in the attenuation, attributed to absorption and/or scattering, are associated with local variations in the viscous properties. Moreover, the mechanical properties appear to show local differences near the weld line compared with areas away from the weld, verifying the spatial-dependent refractive index presented in the THz measurements because the refractive index tends to be higher in a denser medium. Anisotropy also contributes to the contrast [45], as the orientation of fibers in mold polymers were reported to be different within an area close to the weld lines, consequently the spatial variations in the velocities of the different polarizations result in a corresponding variation in the reflectivity; however, probing directional dependencies caused by anisotropy can be done with SAM only when using a cylindrical acoustic lens, which was not the case in this research. 
To conclude this section, we note that $\mathrm{THz}$ surface morphologic measurements, $\mathrm{THz}$ birefringence, and SAM all indicate features consistent with injection-mold weld lines through ROIs I, II, and III at the same locations.

\section{CONCLUSION}

We have carried out $\mathrm{THz}$ imaging and SAM to injection-mold weld lines in an ABS electrical receptacle plate. $\mathrm{V}$-shaped notches are found in the surface morphology by $\mathrm{THz}$ imaging indicating weld lines with a width of $400 \mu \mathrm{m}$ and depth of $\sim 10 \mu \mathrm{m}$. The weld-line locations are confirmed by the observation of line-like features seen at the same locations in SAM of width $500 \mu \mathrm{m}$. In addition, $\mathrm{THz}$ birefringence close to the weld lines indicates the enhanced $\mathrm{THz}$ anisotropy at these locations tentatively assigned to frozen-in molecular orientation and internal stress associated with freezing of the melt due to the fountain flow, which is also supported by the observation of heterogeneity surface in SAM results. The birefringence drops moving away from the weld lines with the material exhibiting more THz isotropy. The locations of these features are consistent with the injection conditions and with mechanical failure under stress.

In conclusion, $\mathrm{THz}$ imaging has demonstrated its ability to detect the presence and spatial extension of anomalies associated with injection-mold weld lines in the nominally amorphous thermoplastic ABS, and the corresponding THz results are supported by SAM measurements and mechanical failure tests. This work suggests that $\mathrm{THz}$ techniques may provide useful means to characterize and locate injection-weld lines in thermoplastic components and potentially this information can be used to improve manufacturing and for the NDE of components on the production line and in service.

\section{ACKNOWLEDGEMENTS}

This work was supported by Conseil Régional Grand Est and CPER SusChemProc. 


\section{REFERENCE}

[1] Fellahi S, Meddad A, Fisa B, Favis BD. Weld lines in injection-molded parts: a review. Adv. Polym. Technol. 1995; 14(3):169-95.

[2] Kim SG, Suh NP. Performance prediction of weld line structure in amorphous polymers. Polym. Eng. Sci. 1986; 26(17):1200-6.

[3] Zhou H, Li D. Modelling and prediction of weld line location and properties based on injection mounding simulation. Int. J. Mater. Prod. Tec. 2004;21(6):526-38.

[4] Tomari K, Tonogai S, Harada T, Hamada H, Lee K, Morii T, Maekawa Z. The V-notch at weld lines in polystyrene injection molding. Polym. Emg. Sci. 1990;30(15):931-6.

[5] Chen CS, Chen TJ, Chien RD, Chen SC. Investigation on the weldline strength of thinwall injection molded ABS parts. Int Commun Heat Mass. 2007; 34(4): 448-55.

[6] Ciubotariu V, Brabie G. Weld line behaviour during uniaxial tensile testing of tailor welded blanks. Arch. Civ. Mech. Eng. 2011; 11(4): 811-24.

[7] Kagitci YC, Tarakcioglu N. The effect of weld line on tensile strength in a polymer composite part. Int J of Adv Manuf Tech. 2016; 85(5-8): 1125-35.

[8] Wu CH, Liang WJ. Effects of geometry and injection-molding parameters on weld-line strength. Polym. Eng. Sci. 2005;45(7):1021-30.

[9] Wang Y, Sun Y, Lv P, Wang H. Detection of line weld defects based on multiple thresholds and support vector machine. NDT\&E Int. 2008; 41(7): 517-24.

[10] Baradi MB, Cruz C, Riedel T, Régnier G. Mechanical and microstructural characterization of flowing lines in injection-molded short fiber-reinforced PBT. Polym. Test. 2019;74: 152-62. 
[11] Tosello G, Gava A, Hansen HN, Lucchetta G, Marinello F. Characterization and analysis of weld lines on micro-injection mounded marts using atomic force microscopy (AFM). Wear 2009;266(5-6):534-8.

[12] Selden R. Effect of processing on weld line strength in five thermoplastics. Polym. Eng. Sci. 1997;37(1) : 205-18.

[13] Bonten C, Tuechert C. Testing of weld joints in Grewell DA, Benatar A, Park JB (Ed.), Plastics and composites welding handbook (pp. 361-392), Hanser München, 2003.

[14] Tadmor Z. Molecular orientation in injection molding. J. Appl. Polym. 1974; 18(6): 1753-72.

[15] Mavridis H, Hrymak AN, Vlachopoulos J. Transcient free-surface flows in injection mold filling. AlChE J. 1988; 34(3): 403-10.

[16] Nguyen-Chung T, Christa P, Gunter M. Flow disturbance in polymer melt behind an obstacle. Rheol Acta. 1998; 37(3): 299-305.

[17] Isayev AI, Shyu GD, Li CT. Residual Stresses and Birefringence in Injection Molding of Amorphous Polymers: Simulation and Comparison with Experiment. J. Polymer Sci.: Part B: Polymer Phys. 2006; 44(3): 622-39.

[18] Chen SC, Chen YC, Cheng NT, Huang MS. Simulation of injection-compression moldfilling process. Intern Comm Heat Mass Transfer. 1998; 25(7): 907-17.

[19] Han J, Shen C, Liu C, Wang S, Chen J. Flow induced birefringence of weldline region in polystyrene injection molding. J Chem Ind Eng. 2008; 59(5): 1305.

[20] Wang Y, Miao Q, Guo Y, Niu C. Research on flow behavior of flat products weld line area amorphous polymer. New Technol \& New Process 2012; 8: 32.

[21] Guo X, Isayev AI. Residual stresses and birefringence in injection molding of semicrystalline polymer: Part II: Experiment and simulation. Intern Polym Process.1999; 14(4): 387-98. 
[22] Wietzke S, Jansen C, Krumbholz N, Peters O, Vieweg N, Jordens C, Scheller M, Romeike D, Jung T, Reuter M, Chatterjee S. Terahertz spectroscopy: A powerful tool for the characterization of plastic materials, In 2020 10th IEEE international Conference on Soild Dielectrics, pp.1-4, IEEE, 2010.

[23] Piesiewicz R, Jansen C, Wietzke S, Mittleman D, Koch M, Kurner T. Properties of building and plastic materials in THz range. J. Infrared Millim. Te. 2007; 28(5): 363 71.

[24] Kraus E, Kremling S, Baudrit B, Heidemeyer P, Bastian M, Stoyanov OV, Starostina IA. Reflection time domain terahertz system for testing polymeric connections. J. Polym. Res. 2015;9(3):345.

[25] Stoik CD, Bohn MJ, Blackshire JL. Nondestructive evaluation of aircraft composites using transmissive terahertz time domain spectroscopy. Opt. Express. 2008; 16(21): $17039-51$.

[26] Federici JF, Schulkin B, Huang F, Gary D, Barat R, Oliveira F, Zimdars D. THz imaging and sensing for security applications-explosives, weapons and drugs. Semicond. Sci. Technol. 2005; 20(7): S266.

[27] Mohamed ET, Zhai M, Schneider G, Kalmar R, Fendler M, Locquet A, Citrin DS, Declercq NF. Scanning acoustic microscopy investigation of weld lines in injectionmolded parts manufactured from industrial thermoplastic polymer. Micron. 2020;138: 102925.

[28] Van Exter M, Fattinger C, Grischkowsky D. Terahertz time-domain spectroscopy of water vapor. Opt. Lett. 1989; 14(20): 1128-30.

[29] Harde H, Cheville RA, Grischkowsky D. Terahertz studies of collision-broadened rotational lines. J. Phys. Chem. A. 1997; 101(20): 3646-60. 
[30] Landau LD, Lifshitz EM. Theory of Elasticity (Course of Theoretical physics). 3d ed. Edited by S. \&T. Books. Oxford: Butterworth-Heinemann, 1995.

[31] Piccarolo S, Saiu M. Knit-line structure in the injection molding of polystyrene. Plast Rub Proc Appl. 1988; 10(1): 11-6.

[32] Malguarnera SC. Weld lines in polymer processing. Polym-Plast Technol. 1982; 18(1): $1-45$.

[33] Nguyen-Chung T. Flow analysis of the weld line formation during injection mold filling of thermoplastics. Rheol. Acta. 2004;43(3):240-5.

[34] Wimberger-Friedl R. The assessment of orientation, stress and density distributions in injection-molded amorphous polymers by optical techniques. Prog Polym Sci. 1995; 20(3): 369-401.

[35] Masanori M, Keiichi N, Takehiro Y, Noriyasu M, Kiyoji N. Welding flow of low density polyethylene melt. J Text Eng. 2003; 49(1): 14-22.

[36] Oh GH, Jeong JH, Park SH, Kim HS. Terahertz time-domain spectroscopy of weld line defects formed during an injection moulding process. Composites Sci. Technol. 2018; 157: 67-77.

[37] Zhai M, Locquet A, Citrin DS. Pulsed THz Imaging for Thickness Characterization of Plastic Sheets. NDT\&E Int. 2020; 116: 102338.

[38] Chen YC, Chen $\mathrm{CH}$, Chen SC. Effects of processing conditions on birefringence development in injection molded parts. II. Experimental measurement. Polym Int. 1996; 40(4): 251-9.

[39] Nguyen-Chung T, Plichta C, Mennig G. Flow disturbance in polymer melt behind an obstacle. Rheol. Acta. 1998;37(3):299-305.

[40] Yu Z, Boseck S. Scanning acoustic microscopy and its applications to material characterization. Rev Mod Phys. 1995; 67(4): 863. 
[41] Da Fonseca RJ, Ferdj-Allah L, Despaux G, Boudour A, Robert L, Attal J. Scanning Acoustic Microscopy-recent applications in materials science. Adv. Mater. 1993; 5(78): 508-19.

[42] Zhang GM, Harvey DM, Braden DR. Microelectronic package characterisation using scanning acoustic microscopy. NDT \& E Int. 2007 Dec 1;40(8):609-17.

[43] Bauermann LP, Mesquita LV, Bischoff C, Drews M, Fitz O, Heuer A, Biro D. Scanning acoustic microscopy as a non-destructive imaging tool to localize defects inside battery cells. J Power Sources Advances. 2020; 6: 100035.

[44] Lemons RA, Quate CF. Acoustic microscope-Scanning version. Appl Phys Lett. 1974; 24(4): 163-5.

[45] Somekh MG, Briggss GA, Ilett C. The effect of elastic anisotropy on contrast in the scanning acoustic microscope. Philos Mag A. 1984; 49(2): 179-204. 


\section{FIGURE}

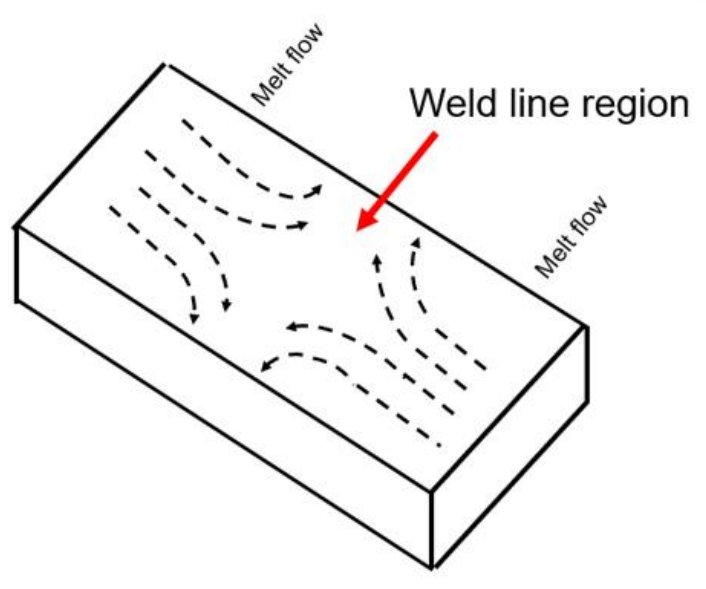

(a).

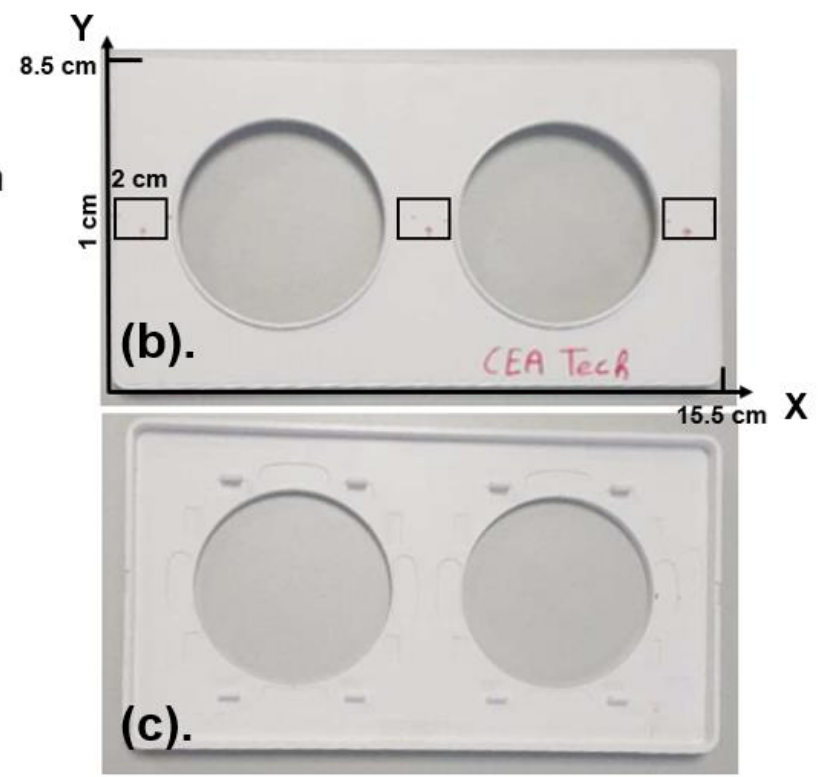

Fig. 1. (a) Schematic diagram of the generation of a stagnating weld line due to fountain flow. Optical photographs of (b) front and (c) back surface of the injected-molded ABS thermoplastic electrical-receptacle plate. THz imaging and SAM are carried out within the three ROIs marked I, II, and III with the THz or acoustical signal incident from the front side. The injection-mold weld line runs horizontally through these three ROIs, and the small red arrow points to the weld location. Dimensions of plate are $85 \mathrm{~mm}$ (vertical direction), $156 \mathrm{~mm}$ (horizontal direction), and $8 \mathrm{~mm}$ (maximum thickness). 

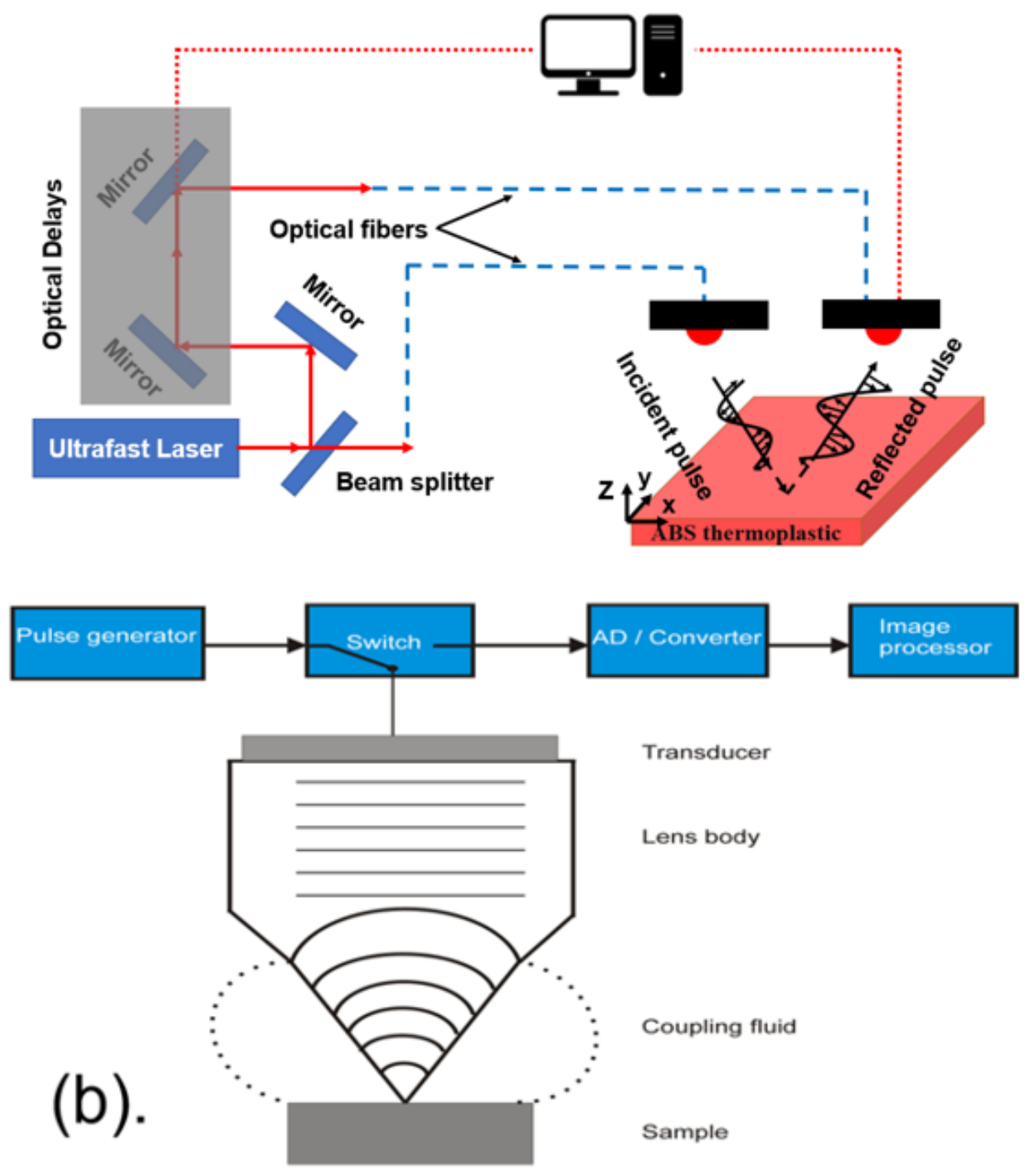

Fig. 2. Schematic diagram of (a) pulsed THz system and (b) SAM. 

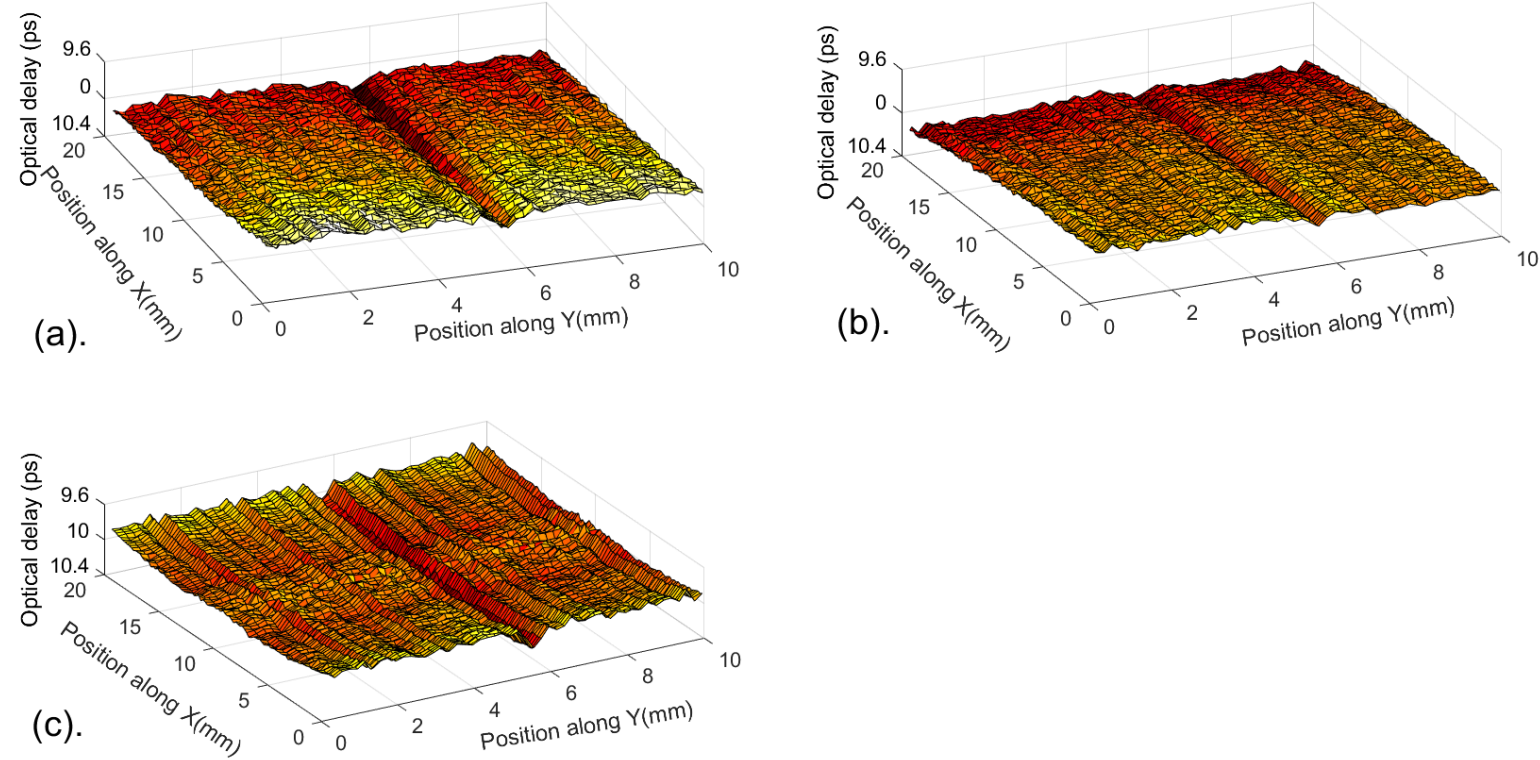

Fig. 3. THz C-scans in ROI (a) I, (b) II, and (c) III based on the peak reflected signal arrival time as the contrast mechanism. The V-shaped notch surface morphological feature at the weld line can be seen clearly for all ROIs. Transverse spatial step size is $200 \mu \mathrm{m}$. The notch width and depth are approximately $400 \mu \mathrm{m}$ and $10 \mu \mathrm{m}$, respectively. 


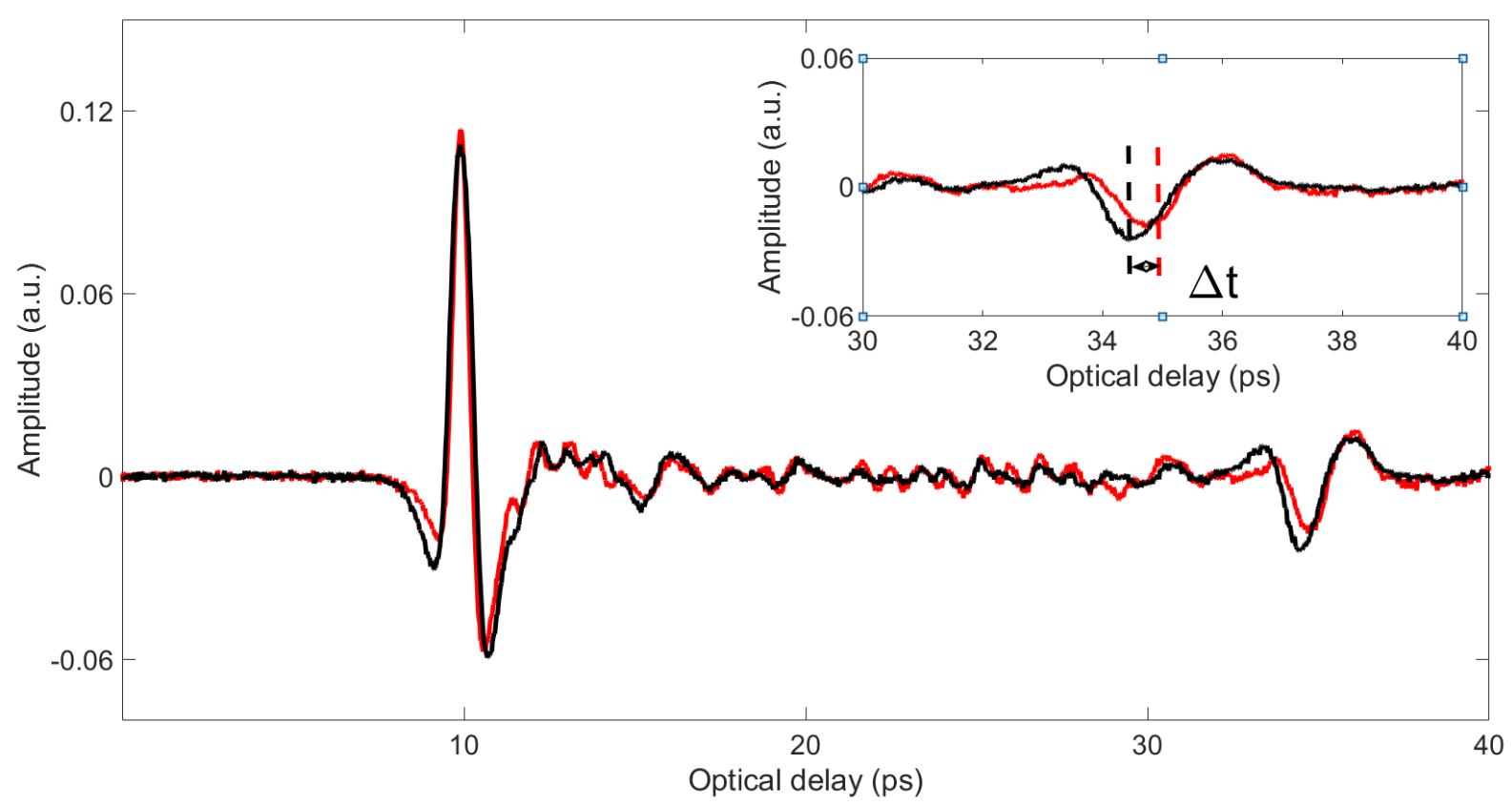

Fig. 4. Typical reflected THz signals from the expected weld line in ROI I with polarization perpendicular (red) and parallel (black) to injection-mold weld line. The first echo at optical delay $\sim 10 \mathrm{ps}$ is due to the reflection at the top air/plastic interface; The second echo at $\sim 35 \mathrm{ps}$ is due to the reflection from the back plastic/air interface. Note that the arrival time of the second echo depends on polarization as is seen more clearly in the inset. The polarization-dependent time delay between the first reflected peak and the reflection from the back plastic/air interface is indicative of the polarization-dependent refractive index of the material near the weld line. 


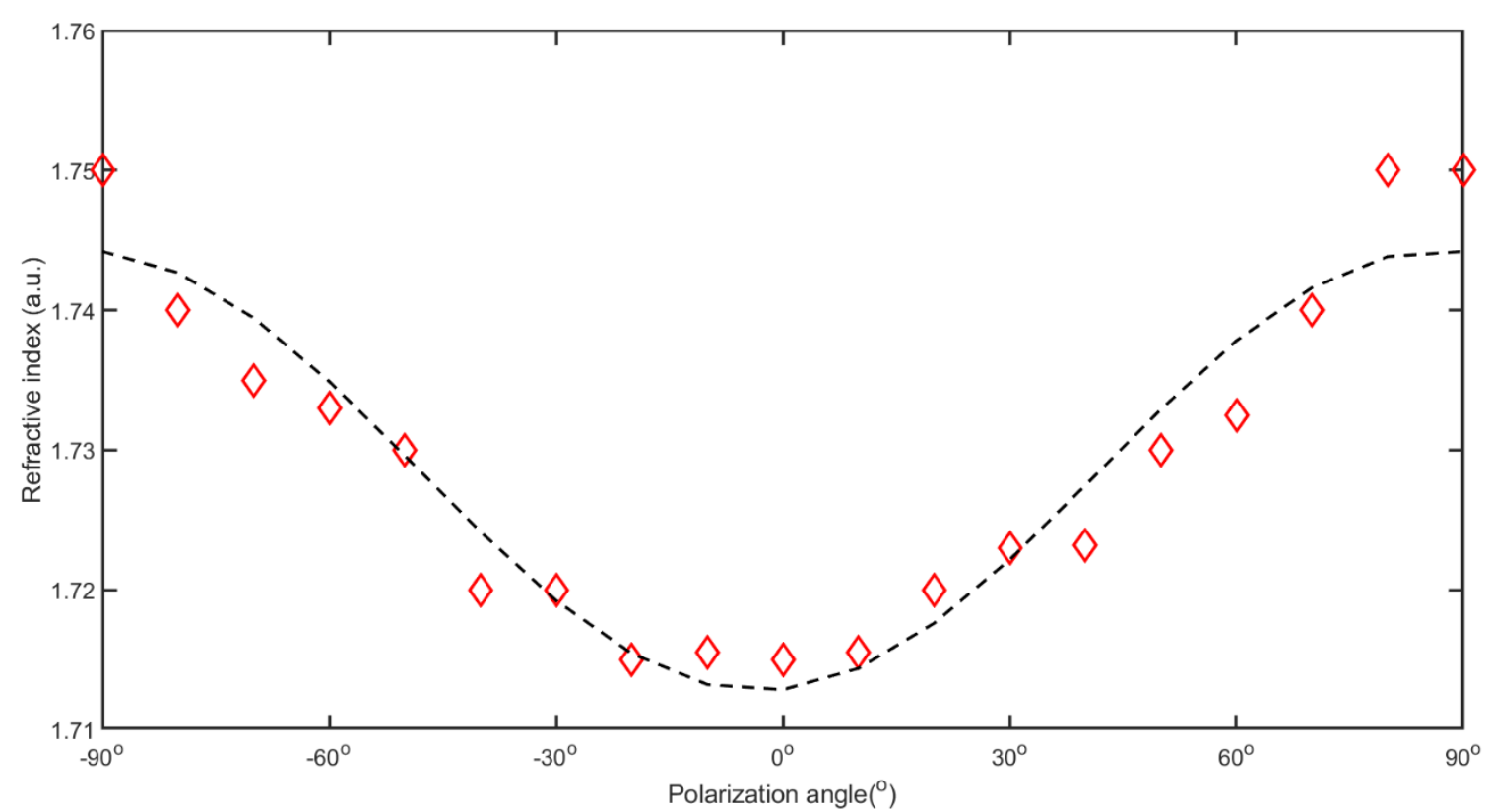

Fig. 5. THz refractive index at weld line as a function of polarization. $-90^{\circ}\left(90^{\circ}\right)$ and $0^{\circ}$ correspond to polarization perpendicular and parallel to the weld line, respectively. Birefringence $\Delta n=0.035$. The corresponding RMSE is 0.003787 . 


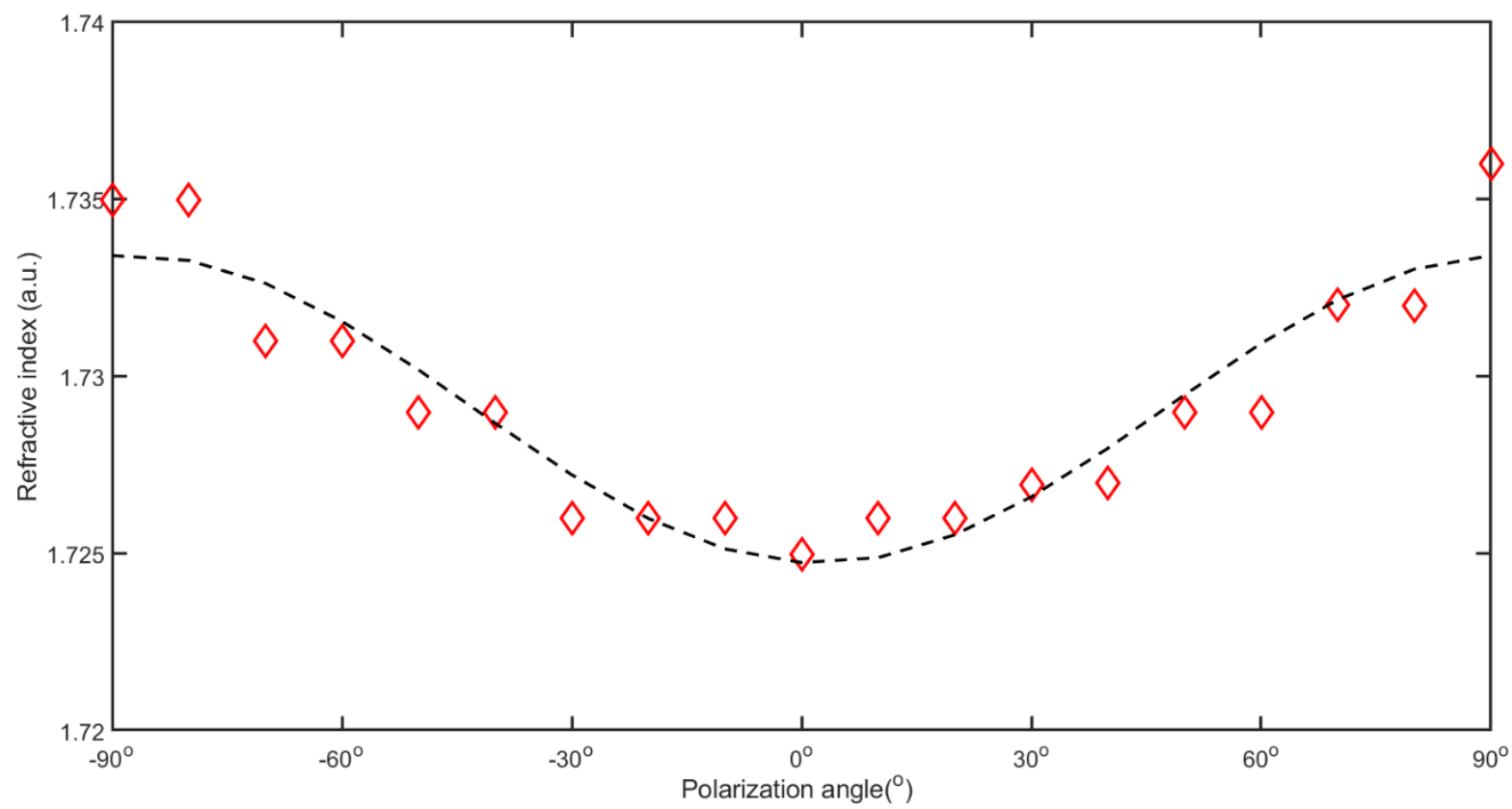

Fig. 6. $\mathrm{THz}$ refractive index $1 \mathrm{~mm}$ away from weld line as a function of polarization. $-90^{\circ}\left(90^{\circ}\right)$ and $0^{\circ}$ correspond to polarization perpendicular and parallel to the weld line, respectively. Birefringence $\Delta n=0.012$. The corresponding RMSE is 0.001287 . 


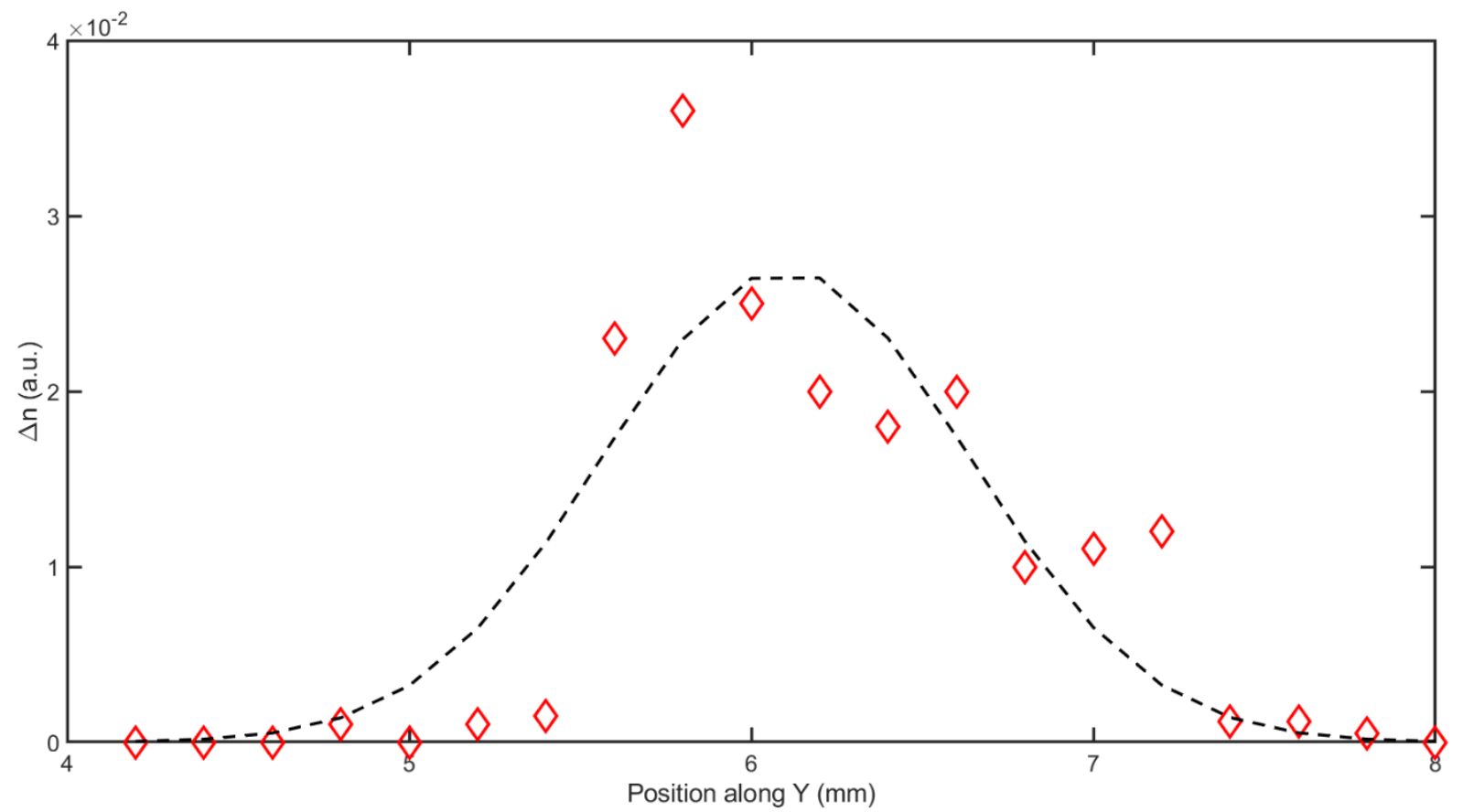

Fig. 7. Birefringence $\Delta n$ along section perpendicular to weld line. Maximum value of $\Delta n$ occurs at the weld line, indicating local enhancement of molecular orientation parallel to the line and frozen-in anisotropic stress. The corresponding RMSE is 0.005507. 

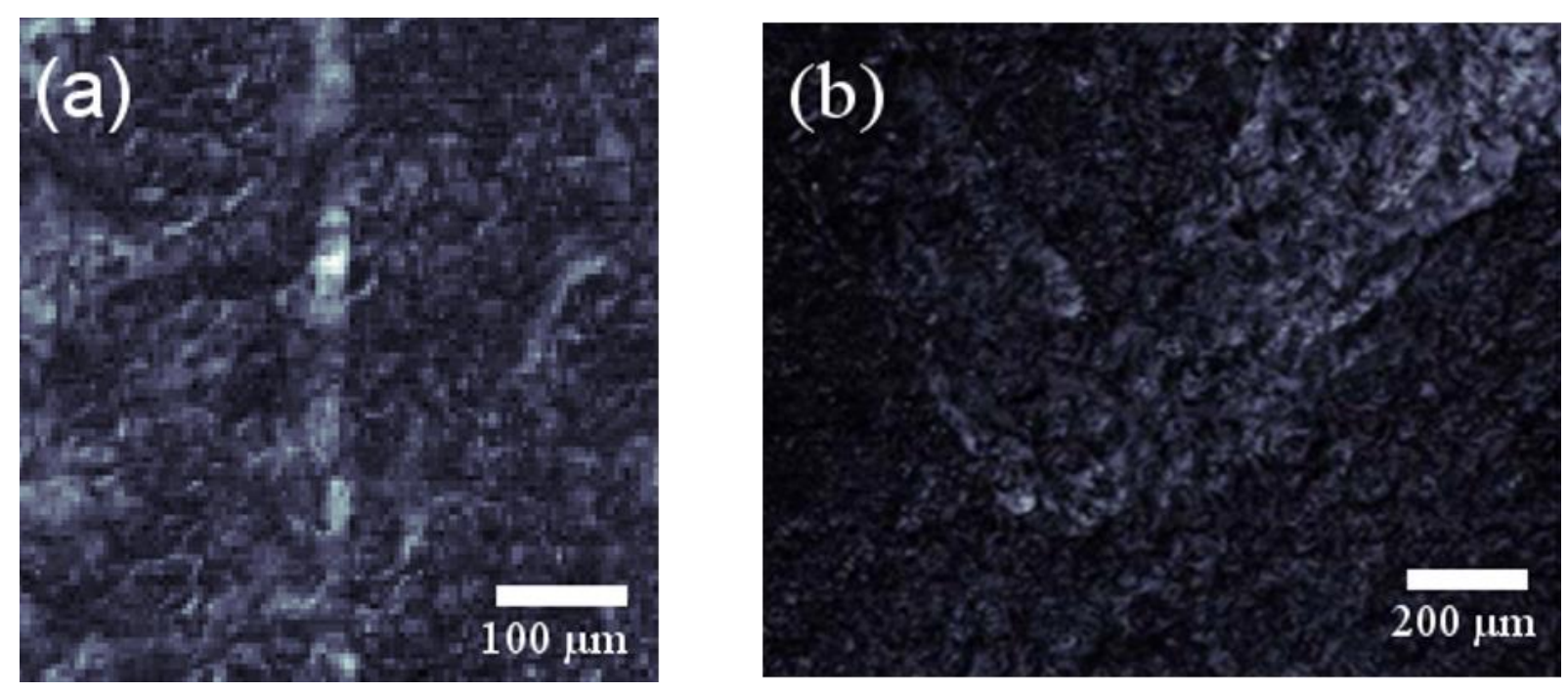

Fig. 8. (a) SAM micrograph of region I of Fig 1 (rotated by $90^{\circ}$ with respect to Fig. 1). The vertical whitish feature is the weld line; irregular flow patterns and surface heterogeneity are also evident. (b) SAM micrograph of a morphological deformation on the surface of the mold about $1 \mathrm{~mm}$ distance from the weld line. Surface heterogeneity is also observable. 